\title{
TURISMO SOSTENIBLE: LUGAREÑOS FRENTE A TURISTAS. EL CASO DE LA CIUDAD DE BARCELONA
}

\author{
M. ABRIL-SELLARÉS ${ }^{1 *}$, M. C. AZPELICUETA² e M. D. SÁNCHEZ-FERNANDEZ ${ }^{3}$ \\ ${ }^{1}$ Universitat Autònoma de Barcelona \\ ${ }^{2}$ Universitat de les Illes Balears \\ ${ }^{3}$ Universidade da Coruña \\ maria.abril@uab.cat
}

Artículo recibido febrero/2015 y aceptó en junio/2015

DOI: $10.15628 /$ holos.2015.2809

\section{RESUMEN}

Este trabajo tiene como objetivo conocer cómo ha afectado el incremento de turistas y sus actividades en su estancia en una ciudad concreta y cómo afectan sus actitudes y acciones a los lugareños de dicha zona. Así mismo se pretende tratar la problemática de los apartamentos turísticos ilegales creados a raíz de una fuerte demanda y las primeras respuestas que ha dado la administración ante las constantes manifestaciones de rechazo de los vecinos de la zona a tratar. La tipología de turismo en la que se centra este estudio es el llamado turismo de masas y concretamente en el turismo de sol y playa y de borrachera, que ha alcanzado altas cotas en estos últimos diez años (Ajuntament de Barcelona 2014), donde los criterios de sostenibilidad parece que no son los principios rectores en la práctica de la actividad turística. Por tanto, hemos visto, por un lado, la necesidad de tratar los conceptos de turismo sostenible y la aplicación que se dan en un caso real: la ciudad de Barcelona y concretamente un barrio marinero como es la Barceloneta y, por otro lado, conocer qué efectos tanto sociales como económicos ha tenido en esta área geográfica concreta la práctica de este turismo.

PALABRAS-CLAVE: Turismo Sostenible, Barcelona, Turistas, Lugareños, Inadaptabilidad

\section{SUSTAINABLE TOURISM: TOURISTS FROM LOCALS. THE CASE OF THE CITY OF BARCELONA}

\begin{abstract}
This work aims to develop know how it has affected the increase in tourists and their activities in its stays in a particular city and how they affected their attitudes and actions to the locals of the area. It also aims to address the problem of illegal tourist apartments created as a result of strong demand and the first answers given by the administration at the continuing demonstrations against the residents of the area to be treated. The type of tourism in which this study focuses is called mass tourism and specifically to the sun and beach tourism
\end{abstract}

and drunkenness, which has reached new heights in the last ten years (Ajuntament de Barcelona, 2014), where sustainability criteria seem to be no guiding principles in the practice of tourism. Therefore, we have seen, at first, the need to address the concepts of sustainable tourism and the application that occur in a real case: the city of Barcelona and specifically a seaside neighborhood as Barceloneta and, moreover, meet how much social and economic effects this has had on specific geographical area where is practicing this tourism.

KEYWORDS: Sustainable Tourism, Barcelona, Tourists, Local people, Inadaptability. 


\section{INTRODUCCIÓN}

En la década de los 80 del siglo XX, a nivel internacional, se ideó un nuevo concepto que se desarrollaría en décadas posteriores: la sostenibilidad que venía marcada por la preocupación por el medio ambiente y la sociedad. Quedaba plasmada dicha inquietud en el llamado informe Brundtland que en su artículo primero definía el desarrollo sostenible como "aquel desarrollo que garantiza las necesidades del presente sin comprometer las posibilidades de las generaciones futuras para satisfacer sus propias necesidades" (COMISIÓN MUNDIAL SOBRE EL MEDIO AMBIENTE Y DESARROLLO, 1987:1). Paulatinamente el concepto de sostenibilidad se fue aplicando a todos los sectores económicos de la sociedad moderna, incluyendo, el turismo, donde autores como CAPECE (1997) y BLASCO (2006) han definido el turismo sostenible entendiéndolo cómo aquel que respeta los límites, tanto físicos de los destinos como psíquicos de los lugareños, teniendo en cuenta los costes, los impactos tanto positivos como negativos así como los riesgos de un turismo poco ajustado al destino al que accede.

Por su parte la llamada Carta de Lanzarote (CANARIAS, 1995) expresaba el hecho de que el turismo es un potente instrumento de desarrollo y como consecuencia de ello asume la necesidad de que participe en la estrategia del desarrollo sostenible. De modo que si se produce una gestión correcta del turismo conllevará la exigencia de garantizar la sostenibilidad de los recursos. Le corresponde a la administración pública tomar las decisiones capaces de alcanzar metas que tengan en cuenta el desarrollo sostenible, protegiendo de este modo a los recursos patrimoniales tanto tangibles como intangibles del territorio que haya que ordenar (BLASCO, 2006). Para tomar decisiones hay que realizar un análisis, que tenga en cuenta, por un lado la capacidad de carga de los recursos patrimoniales en general, la calidad de vida de los turistas a quién irán dirigidas las acciones pero también la de los autóctonos, teniendo en cuenta, en este caso la opinión de los mismos, aplicar el principio de que no siempre la actividad turística puede llegar a ser la mejor opción y finalmente tener presente que las actividades turísticas son interdisciplinarias. (AVILA, 2002).

En el turismo sostenible existen los llamados criterios globales (GLOBAL SUSTAINTABLE TOURISM COUNCIL, 2012), que representan los mínimos a alcanzar por cualquier empresa turística en el ejercicio de su actividad. Estos criterios se organizan en cuatro grandes temas principales: 1- la planificación eficaz para la sostenibilidad. 2- la maximización de los beneficios sociales y económicos para la comunidad local. 3- el mejoramiento del patrimonio cultural. 4- la reducción de los impactos negativos sobre el ambiente. Como puede apreciarse criterios que van referidos a los impactos tanto a nivel económico como social.

En el caso de estudio que aquí nos ocupa: la ciudad de Barcelona y concretamente el barrio de la Barceloneta y la relación vecinos - turistas, nos vamos a centrar en los impactos tantos sociales como económicos. Según Morant (1996), España ha seguido el mismo modelo turístico durante años, atraer el máximo número de visitantes, durante el máximo tiempo posible y que su gasto se centre en el consumo de productos y servicios reduciendo al mínimo los costes fijos de explotación permitiendo con ello aumentar el beneficio empresarial, a menudo en detrimento de los estratos sociales existentes. (MORANT et. al, 1996). Es por ello que cuando se han empezado a tener en cuenta los modelos del turismo sostenible se ha pasado a un modelo donde hay que entender la actividad turística no sólo como un efecto económico sino también 
como un efecto social. (VERA, 2002). De este modo la maximización de los beneficios y la reducción de los impactos negativos son fundamentales para evitar lo que se ha venido llamando en estos últimos tiempos, la "turismofobia", es decir, el rechazo a los turistas por parte de los lugareños.

La ciudad de Barcelona desde los años 90 del siglo XX ha ido incrementando tanto los servicios como los productos turísticos lo que la ha convertido en uno de los destinos urbanos más solicitados a nivel internacional (Ajuntament de Barcelona, 2014). Pero su evolución ha ido planteado problemas tanto a nivel de infraestructuras, como de su sistema productivo y de las preservación de su propia identidad tanto social como cultural, sufriendo con ello unas transformaciones que muy probablemente no se hubieran producido de no haberse constituido en un espacio turístico (BLASCO, 2006).

Es evidente que hay que plantearse los modelos de desarrollo local que mejor se adapten a cada uno de los espacios en los que el turismo afecte directamente. De modo que, si bien hay que "prestar facilidades a las empresas y dejar actuar al mercado dentro de un razonable control social, o planificar la orientación de la actividad económica en conjunto, con el objetivo de incrementar el bienestar de toda la sociedad", como señala (PARDELLAS \& PADíN, 2004: 109) la necesidad de escuchar a los agentes locales (comerciantes, vecinos) y tener clara consciencia de la necesidad de equilibrar el territorio pasan hoy en día a ser claros objetivos del turismo sostenible. El caso de la ciudad de Barcelona, y concretamente del barrio de la Barceloneta, los principios del turismo sostenible se han visto afectados desde dos puntos de vista que afecta a dichos agentes locales: por un lado los alquileres de las viviendas tratándolas como apartamentos turísticos y por otro lado, el equilibrio y respeto a los lugareños que han visto menguar su calidad de vida.

Teniendo en cuenta estas premisas surgió la necesidad de realizar la presente investigación, donde los objetivos son tres: el primero, conocer cuál es el grado de rechazo, el segundo cómo se plasma en la ciudad, y el tercero, cuáles son las medidas correctoras que se han tomado por parte de las instituciones gubernamentales.

\section{METODOLOGIA}

En base a los objetivos anteriormente establecidos, la metodología empleada se ha basado en dos pilares: por un lado, un análisis de la literatura científica que permite argumentar los conceptos y análisis del turismo sostenible y por otro, un vaciado de las noticias en periódicos y páginas web, durante la segunda quincena del mes de agosto de 2014, sobre la problemática social existente en el barrio de la Barceloneta de la ciudad de Barcelona.

Para conocer la problemática social, se han trabajado un total de 77 artículos de carácter nacional e internacional publicados digitalmente entre los días 20 de agosto y 31 de agosto (ambos inclusive). Posteriormente se han clasificado dichos artículos en dos grandes grupos: aquellos que hablaban básicamente sobre los apartamentos turísticos ilegales y aquellos que hablaban de la problemática directa entre lugareño y turista en su día a día.

En el primer grupo, se han identificado los elementos que han provocado el desasosiego en los habitantes del barrio desde el punto de vista básicamente económico: precios abusivos en los alquileres de la vivienda, incremento de precios en las tiendas habituales. Mientras que en el 
grupo segundo, básicamente dedicado a temas sociales, se han observado los siguientes ítems: escasez de higiene, deterioro del entorno, poco respeto a los lugareños, miedo de saber quién entra en el edificio, inseguridad ciudadana, impacto del ruido.

Finalmente, a través de los resultados obtenidos, gracias al análisis de los artículos de prensa digital, se ha podido llegar a la verificación los tres objetivos que nos habíamos planteado al inicio de la investigación

\section{RESULTADOS Y DISCUSIONES}

De los 77 artículos investigados, la máxima preocupación que presentan en sus portadas son:

Tabla 1: Las Portadas de la prensa digital (del 20 al 31 de agosto).

\begin{tabular}{l|c|c}
\hline \multicolumn{1}{c|}{ Prensa } & Número & $(\%)$ \\
\hline - Apartamentos de Alquiler ilegales & 18 & $23,37 \%$ \\
- Turismo de Masa / low Cost & 18 & $23,37 \%$ \\
- Turismo de Borrachera & 36 & $46,74 \%$ \\
- Combinando los ítems anteriores & 5 & $6,49 \%$ \\
\hline
\end{tabular}

Como puede apreciarse por la Tabla 1 hay un mayor impacto en términos de carácter social que de carácter económico. Sólo un $6 \%$ de los artículos en su encabezamiento reflejan la doble problemática vivida durante el mes de agosto en el barrio de la Barceloneta: los pisos de alquiler ilegales y el turismo de masas, low cost y/o borrachera. Mientras, que sumando el turismo de masa/ low cost, entendido como un determinado modelo turístico encontrándose en él el llamado turismo de borrachera, desde hace unos diez años, la cifra de preocupación en los titulares de los artículos alcanza el $69,74 \%$ de los mismos, y sólo un poco más del $23 \%$ muestran en sus titulares la preocupación por temas económicos

\subsection{Respecto al impacto económico}

Los resultados obtenidos respecto a los impactos económicos en el barrio de la Barceloneta son:

- Preocupación por la subida de los alquileres que afectan a las viviendas antiguas. Se señala que por una vivienda de $30 \mathrm{~m}^{2}$ cuyo alquiler medio, en dicho barrio, es de 800 a $1.000 €$ al mes, se pasa a un alquiler turístico entre $1.000 €$ por 10 días y $3.000 €$ al mes

- Algunos lugareños, deciden irse de vacaciones a otros lugares y alquilar sus viviendas para obtener unos réditos y ayudar a la economía familiar, en unos momentos de crisis importantes, lo que provocan situaciones de ilegalidad pues no suelen tener licencia para abrirse como alojamiento turístico.

- Otros son los pisos que buscados por las empresas inmobiliarias provocan las crecidas desmesuradas de los alquileres y que dejan fuera de mercado a residentes que una vez terminado su contrato de alquiler no pueden hacer frente a los nuevos precios que instaura el mercado 
- En definitiva, según los artículos, en la actualidad, clasificados como pisos legales turísticos hay 72 viviendas. Pero en términos de ilegalidad, los pisos de alquiler se mueven entre 800 y 1.000 pisos. Los artículos hacen hincapié que los vecinos señalan tanto de las empresas que están realizando negocios ilícitos como los residentes que alquilan su propia vivienda, indicando que sólo basta con mirar las páginas web para percatarse de lo que está sucediendo.

- Los comerciantes señalan que la llegada de turistas a su zona les ha beneficiado, pues venden más, sus restaurantes se llenan y eso les permite tener unos beneficios que con anterioridad a este boom no tenían. Los vecinos en cambio se quejan del aumento de los precios de la cesta de la compra, y lo achacan en mayor medida a la llegada de los turistas.

Los responsables de la gestión local, por su parte, han adoptado dos medidas: por un lado enviar inspectores para ver qué pisos están alojando turistas de manera ilegal. Los propios vecinos señalan que es una medida innecesaria y que las acciones se están dando porqué hay las manifestaciones. Por otro reunirse con los agentes gestores turísticos de los alojamientos de la ciudad (asociaciones hoteleras, grandes cadenas, pequeños hoteles, apartamentos turísticos legales, etc.) para ver qué medidas se pueden adoptar para poder controlar de manera eficiente el problema de los pisos ilegales, que repercute, evidentemente, en que la capacidad hotelera de la ciudad sea menor.

\subsection{Respecto al impacto social}

Palabras como incivismo, borrachera, poco respeto, ruido, suciedad, antisociales, descanso y desnudez son las que afloran del vaciado de los artículos. Así tenemos

Tabla 2: Los sustantivos que conducen a la insostenibilidad.

\begin{tabular}{l|c}
\hline \multicolumn{1}{c|}{ Prensa } & Número de veces que aparecen \\
\hline - Incivismo & 66 \\
- Turismo de Borrachera & 202 \\
- Irrespetuosos & 18 \\
- Ruido & 22 \\
- Suciedad & 20 \\
- Antisociales & 3 \\
- No descanso & 27 \\
- Desnudez & 34 \\
- Insostenible & 15 \\
- Horda & 4 \\
\hline
\end{tabular}

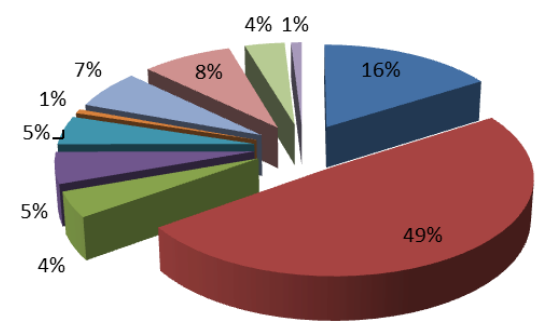

$$
\begin{aligned}
& \square \text { - Incivismo } \\
& \square \text { - Turismo de Borrachera } \\
& \square \text { - Irrespetuosos } \\
& \varpi \text { - Ruido } \\
& \square \text { - Suciedad } \\
& \square \text { - Antisociales } \\
& \varpi \text { - No descanso } \\
& \square \text { - Desnudez } \\
& \square \text { - Insostenible } \\
& \square \text { - Horda }
\end{aligned}
$$

Figura 1: Porcentajes de los sustantivos que conducen a la insostenibilidad 
Es evidente que lo que más preocupa a los lugareños y que aparece reflejado en los artículos en más de una ocasión (véase Tabla 2 y Figura 1) es el turismo de borrachera, ya que de ella se desprenden muy probablemente los ruidos nocturnos, la suciedad, el no poder descansar y la falta de respeto. Además una de las preocupaciones es el carácter incívico que muestran algunos turistas y que preocupa al ciudadano y que se ve reflejado en ese $16 \%$ de llamadas al sustantivo que se han hecho en los artículos.

Estas actitudes han conllevado a los lugareños a manifestarse cada día por la tarde-noche frente a las instituciones administrativas locales e incluso autonómicas pidiendo poner remedio a una situación que en su barrio se ha hecho insostenible. Y aún más, que en las últimas manifestaciones se han añadido lugareños de otros barrios con lo que se corre el riesgo, que esta llamada de alerta por parte de unos ciudadanos, acabe convirtiéndose en un problema para toda la ciudad.

Es curioso observar el uso de la palabra horda. Los artículos de los periódicos la utilizaban entrecomillada ya que eran los comentarios que los vecinos hacían cuando intentaban definir algunas de las actitudes y acciones incívicas de los turistas, un "grupo de gente que obra sin disciplina y con violencia”, según la (RAE, 1950:835). De momento la acción sin disciplina es clara, gente desnuda por la calle, o en bikini, vomitándo en cualquier punto, cantando y gritando pasadas las diez de la noche, entre otras acciones, que están reguladas y sancionadas por las Ordenazas de la ciudad de Barcelona

Finalmente las palabras "basta" y "hartos" ha salido tanto para el impacto económico como para el impacto social, haciéndose eco los periódicos de cómo las han utilizado los lugareños cuando han expresado su malestar: en pancartas colgadas de los balcones, en dibujos en las aceras o en carteles publicitarios y finalmente en las pancartas de las manifestaciones.

Las acciones emprendidas por los responsables públicos locales ha sido básicamente, tal y como señalan los periódicos, aumentar el número de policías que se pasea por la zona, con el fin de explicar a los turistas que con esa actitud no se va a ninguna parte, siendo pocas veces la acción coercitiva la que se ha llevado a cabo y menos aún el multar la acción que se tipifica en las Ordenanzas municipales. Se quejan los lugareños, que hay dos varas de medir y que en Barcelona no vale todo, ni que el barrio está en venta, dos frases con las que se ha cerrado más de un artículo analizado, en concreto en 23 ocasiones.

\section{CONCLUSIONES}

Los resultados de este estudio permiten verificar los tres objetivos que nos habíamos planteado inicialmente. Por un lado, el grado de rechazo de los lugareños, es alto, en menos de 15 días, 77 artículos de periódico se hicieron eco del malestar ciudadano y de su expresión a través de escritos y manifiestos. Es bien cierto, que este grado de malestar ha sido un hecho puntual en la ciudad, pero pone en alerta a los responsables y gestores turísticos para conseguir un mejor plan de acción turístico donde el concepto de sostenibilidad no se quede sólo en el marco teórico.

En segundo lugar, los lugareños han expresado su malestar, colgando en las redes sus inquietudes: fotos, pancartas, acciones que muestran hasta qué punto ha llegado su indignación, 
prueba de ello es el volumen de artículos publicados no sólo de referente nacional sino también extranjero

Y en tercer lugar, Las nuevas propuestas turísticas de la ciudad de Barcelona tienen que estar en consonancia con la sostenibilidad, para ello los responsables de la gestión turística han de controlar a las empresas y a los residentes que de manera privada están obrando al margen de la ley. El Ayuntamiento de la ciudad ha iniciado ya algunas acciones a través de inspecciones y de alertar a los turistas de que ciertas acciones no son acordes con las Ordenanzas municipales.

En definitiva la sostenibilidad turística, en un área de la ciudad de Barcelona, ha visto cómo se tambaleaban sus principios cuando los lugareños han emprendido una serie de acciones frente no sólo las actitudes y acciones de los turistas sino también de aquellos agentes empresariales y personas privadas que en aras de obtener unos beneficios perjudican al resto de la comunidad residente. Es un hecho puntual, en una zona concreta, pero hay que tenerlo presente, la primera alarma ya ha saltado hay que evitar con planes y gestión municipal correcta que se extienda y acabe creando una fractura social de difícil solución.

\section{REFERENCIAS BIBLIOGRÁFICAS}

1. AJUNTAMENT DE BARCELONA. Ajuntament de Barcelona, notícies, 29 ago. 2014. Disponível em: <http://w110.bcn.cat/portal/site/Turisme/menuitem.7e257cdd389697aa58cb0d302034 8a0c/?vgnextoid=f55e320bf2652410VgnVCM1000001947900aRCRD\&vgnextchannel=00000 00602804218VgnV6CONT00000000000RCRD\&vgnextfmt=formatDetall\&lang=es_ES>. Acesso em: 12 set. 2014.

2. AVILA, R. Turismo sostenible. Madrid: lepala, 2002.

3. BLASCO, A. Tipologías turísticas. Girona: Universitat de Girona, 2006.

4. CAPECE, G. Turismo sostenible y sustentable. Una visión holística. Buenos Aires: Edición Cid, 1997.

5. COMISIÓN MUNDIAL SOBRE EL MEDIO AMBIENTE Y DESARROLLO. Nuestro Futuro Común. Nueva York: United Nations, 1987.

6. ELCOMERCIO. Barcelona estalla contra el turismo de borrachera. Barcelona, España, 21 ago. 2014

7. GLOBAL SUSTAINTABLE TOURISM COUNCIL. Criterios Globales de Turismo Sostenible para Destinos, 2012. Disponível em: <http://Criterios\%20Globales\%20de\%20Turismo\%20Sosteni ble\%20-\%20Destinos\%20_borrador\%202.pdf>. Acesso em: 25 out. 2014.

8. MORANT, A.E. Oferta Turística. In: PEDREÑO, A. (Dir) Introducción a la economía del turismo en España. Madrid: Civitas, 1996.

9. PARDELLAS, X., PADÍN, C. Una propuesta de turismo sostenible para el municipio de Caldas de Rei (Pontevedra). Cuadernos de Turismo, p. 107-125, 2004.

10. RAE. Diccionario manual e ilustrado de la lengua española. Madrid: Espasa-Calpe, 1950.

11. VERA, F. Estrategias de diversificación y diferenciación en destinos turísticos litorales: la revalorización del patrimonio cultural urbano". In: Pardellas, X. Estrategías turísticas urbanas. Vigo: Universidad de Vigo, 2002. 